Continents manuscrits

Génétique des textes littéraires - Afrique, Caraïbe, diaspora

2 | 2014

Bien écrire, mal écrire

\title{
Mot muet crie
}

\section{L'écrivain Raoul Hausmann}

Adelheid Koch-Didier

\section{(2) OpenEdition}

12 Journals

Édition électronique

URL : http://journals.openedition.org/coma/299

DOI : $10.4000 /$ coma.299

ISSN : 2275-1742

Éditeur

Institut des textes \& manuscrits modernes (ITEM)

Référence électronique

Adelheid Koch-Didier, "Mot muet crie », Continents manuscrits [En ligne], 2 | 2014, mis en ligne le 22 avril 2014, consulté le 21 avril 2019. URL : http://journals.openedition.org/coma/299 ; DOI : 10.4000/ coma.299

Ce document a été généré automatiquement le 21 avril 2019

\section{(c) (i) (9)}

Continents manuscrits - Génétique des textes littéraires - Afrique, Caraîbe, dispora est mis à disposition selon les termes de la licence Creative Commons Attribution - Pas d'Utilisation Commerciale - Pas de Modification 4.0 International. 


\title{
Mot muet crie
}

\author{
L'écrivain Raoul Hausmann
}

\author{
Adelheid Koch-Didier
}

\author{
Mot \\ muet \\ crie \\ noir \\ sur blanc \\ mort veut crier \\ crier dans le monde ${ }^{1}$.
}

1 «J'invente des mots et la syntaxe, ce que l'on appelle barbarisme, mais mes barbarismes en allemand furent déjà décisifs dès $1918^{2}$. » C'est ainsi que se défend Raoul Hausmann (Vienne 1886 - Limoges 1971), à propos de La Sensorialité excentrique (1970), son ouvrage en quelque sorte testamentaire. Co-fondateur du dadaïsme à Berlin (1918), le surnommé « dadasophe officiel » est encore trop peu perçu comme écrivain de langue française. Ses archives conservées au musée d'Art contemporain de Rochechouart près de Limoges permettent cependant de découvrir cet artiste protéiforme - peintre, photographe, poète, romancier, théoricien, danseur- qui s'attribuait à juste titre l'invention de « 5000 paroles et de 10000 formes $^{3}$ ».

2 À partir du moment où Hausmann, depuis 1933 en émigration quasi permanente, veut ou doit s'imposer comme auteur français, sa «nationalité littéraire », relevant du choix de la langue de création, devient au moins double. Car s'il est vrai qu'il écrit, à partir de son installation définitive en France en 1944, plus de textes en français qu'en allemand, il n'en reste pas moins que beaucoup de ses poèmes se situent volontairement « entre poème phonétique et poème presque allemand ", qu'ils oscillent entre poème phonétique et presque français ou encore entre «poèmes en trois langues » et véritables «languemélanges ", pour ne reprendre que deux titres de ses textes ${ }^{4}$. Bon nombre de ses écrits poétiques se produisent en fonction de jeux de mots et calembours, des permutations phonétiques et syntaxiques, des glissements sémantiques et métaphoriques. Ainsi, ils jettent d'une part le pont entre la poésie de langue allemande et celle de langue française; d'autre part, ils transgressent délibérément les systèmes linguistiques jusqu'à la poésie phonétique pure, 
dépourvue de tout apanage sémantique, dont le but suprême est la coïncidence du son, de l'écriture et de l'image.

3 Il n'empêche que l'inventeur du poème "optophonétique ", précurseur de la poésie dite «lettriste », court le risque de se voir reprocher non seulement son allemand « torturé ${ }^{5}$ ", mais aussi et surtout, sa connaissance insuffisante du français. "À mon humble avis ", estime en 1942 un rédacteur des Cahiers du Sud dans une lettre à Hausmann, «je crois [que] vous avez tort de vouloir écrire directement en français une œuvre originale ${ }^{6} » . \mathrm{Ce}$ jugement impitoyable en dit long, non seulement sur la maîtrise imparfaite qu'il a pu avoir à cette époque de l'écriture et de la pensée françaises, mais aussi sur les difficultés auxquelles un auteur de langue allemande émigré en France devait s'attendre. Plus encore un dadaïste en Limousin qui « tourne et retourne pendant toute la journée tous les mots ", qui « parle en français, anglais et allemand, et en dada $^{7}$ »!

4 Notre article se propose de retracer le lien entre le multilinguisme de Hausmann et sa créativité d'écrivain qui s'obstine à s'affranchir de toutes les normes linguistiques et s'efforce de gommer les clivages entre les langues et langages, entre les différents modes d'expression, entre la littérature et les arts plastiques. Pour ce faire, nous suivrons dans un premier temps le fil biographique pour arriver dans un second temps à la description de trois textes majeurs en langue française avant de présenter plus en détail les archives conservées en France, des éditions posthumes et des trésors qui restent encore à exploiter.

5 Né à Vienne le 12 juillet 1886 de parents autrichiens, Hausmann est, selon une définition que Richard Thieberger donne au sujet de la notion controversée d'« écrivain autrichien ", sans aucun doute un "authentique Autrichien ${ }^{8}$ ». Les origines de la famille s'avèrent pourtant - et précisément pour cela - beaucoup plus complexes : « Ma famille paternelle s'était établie en Tchécoslovaquie en 1809. Mes aïeux étaient de Strasbourg, malgré cela, toute ma famille paternelle et maternelle était d'origine tchèque ou slovaque, ou même italienne9."

6 Hausmann ne passe que les quatorze premières années de sa vie dans son pays natal et n'y retourne jamais ${ }^{10}$. En 1900, après un bref séjour à Munich, la famille s'installe à Berlin parce que Victor Hausmann, le père de Raoul, est engagé comme peintre académique à la cour de Guillaume II $^{11}$.

7 Très attaché à ses origines autrichiennes, plus exactement à ses ancêtres tchécoslovaques dans les confins de l'ancienne monarchie austro-hongroise, Hausmann adopte une position radicalement anti-allemande. Dans sa correspondance avec Tristan Tzara de même que dans une «Lettre ouverte » à l'Ambassade tchécoslovaque à Paris ${ }^{12}$, «le plus grand expérimentateur d'Autriche ${ }^{13} »$ - comme il se définissait lui-même - ne cesse de souligner son malaise d'être considéré comme Allemand. $\mathrm{Au}$ lieu de s'appeler Hausmann - «Ton nom Hausmann, est boche »- il aurait préféré «dans les champsélysées de l'Esprit », se nommer "Nnamsuah", comme si l'inversion du patronyme suffisait pour changer d'identité.

8 La nostalgie de ses origines tchécoslovaques devient non seulement le moteur de sa critique de l'Allemagne, exprimée entre autres dans les douze satires politiques Hurrah! Hurrah! Hurrah! $!^{14}$, mais décidera de sa vocation de poète, à en croire le texte autobiographique Dadasophie ${ }^{15}$, dans lequel il se rappelle l'effet produit sur lui par une chanson folklorique tchèque que son père avait l'habitude de chanter :

À la haie nous étions dressés, nous avons ri, à la haie, et nous avons ri dressés à la haie où nous étions dressés en riant et nous avons ri, à la haie dressés, dressés à la haie où nous avons ri, nous avons ri à la haie dressés en riant, tous les deux ${ }^{16}$. 
Cette petite chanson peut sembler insignifiante ou insensée, aux yeux du jeune Raoul elle est même presque bête. Et pourtant s'en dégagent deux tendances fondamentales de sa poésie. L'une est formelle : la répétition sérielle et variée ; l'autre thématique : l'écho d'un formidable éclat de rire. Le rire provocateur deviendra, comme on le sait, l'arme la plus puissante du mouvement Dada en butte à la société et à l'art bourgeois.

10 Une deuxième impulsion lui vient, d'après ses souvenirs dans Dadasophie, du nom du petit village tchèque de Stehelceves, près de Prague, où père et fils Hausmann avaient droit de cité, nom qui signifie en français «chant d'oiseaux » ou plus fidèlement «chant du chardonneret ». S'en inspire le poème Oiseautal ${ }^{17}$, écrit probablement en 1947, mais antidaté de 1919, sans doute le poème le plus onomatopéique de Hausmann, imitant parfaitement le chant naturel des oiseaux. Partant du modèle littéraire Appel des oiseaux du poète grec Aristophane, il répond en même temps à la Superchanson d'Oiseau (1946) de son ami et collègue Kurt Schwitters.

11 La poésie phonétique et « optophonétique » que Hausmann ne cesse de pratiquer jusqu’à la fin de sa vie se situe, à vrai dire, en deçà du bien ou du mal écrire. Elle est au-delà des normes et critères de jugement habituels et se décline sur différents supports: manuscrits, dactylographies, dessins, feutres, toiles. Lettres-signes et Signes-écriture et d'autres œuvres conservées au MAC donnent à voir le dépassement du mot vers l'image et de la linéarité vers la spatialité.

12 Comme la plupart des intellectuels allemands et autrichiens, Hausmann arrive en France en 1938, de Prague, à la suite de l'occupation de la Tchécoslovaquie par les troupes d'Hitler. La France était la dernière étape de son «émigration permanente ${ }^{18}$ ", comme Alfred Kolleritsch qualifie à juste titre l'itinéraire mouvementé de Hausmann: Vienne, Berlin, Ibiza, Zurich, Prague, Paris - pour ne citer que les étapes les plus importantes de ses pérégrinations de 1886 à 1938.

13 À Paris, il gagne péniblement sa vie avec des travaux photographiques et la publication d'articles sur l'architecture rurale méditerranéenne. En septembre 1939, à l'éclatement de la Seconde Guerre mondiale, Hausmann, encore citoyen tchécoslovaque ${ }^{19}$, n'a pas à redouter l'internement au même degré que les autres émigrants allemands, mais il juge tout de même indispensable de quitter Paris. Il se réfugie dans la zone libre, à Peyrat-leChâteau dans le département de la Haute-Vienne où s'était formée une petite colonie d'artistes en exil. Après la Libération, il s'installe en novembre 1944 à Limoges où il reste jusqu'à sa mort en 1971; au seuil de la soixantaine, il y commence une œuvre tardive foisonnante.

14 La reprise de l'activité artistique après 1945 en France ne se limite pas à assurer et à revaloriser les acquis de l'époque Dada tels que la peinture abstraite, la photographie expérimentale et le photomontage, le collage et le poème (opto-) phonétique. Simultanément, Hausmann entre dans une nouvelle phase de sa création et s'investit énormément dans l'élaboration de textes en français. Contrairement aux émigrés autrichiens restés dans les pays d'exil et écrivant, dans un environnement linguistique étranger, leurs livres en allemand, Hausmann continue non seulement de se servir de sa langue maternelle, mais adopte le français comme mode d'expression privilégié ; il suffit de comparer le nombre de livres que Hausmann rédige en allemand et en français ou encore les poèmes écrits dans les deux langues pour se convaincre de la prédominance du français après $1945^{20}$. Bien évidemment, ce choix correspond dans une large mesure à l'espoir - pas toujours satisfait - de trouver plus facilement des éditeurs. 
Les années qui suivent l'installation de Hausmann en France sont en majeure partie consacrées au genre dramatique au sens large du terme. Pièces de théâtres, dialogues farfelus, pantomimes sanglantes et représentations scéniques se succèdent. Le meurtre prodigieux, L'amour jusqu'à la mort, La mort de la vierge et d'autres titres annoncent un thème omniprésent, pour ne pas dire obsédant, celui de la mort, de la disparition anormale, criminelle ou absurde.

16 À la fin des années cinquante, Hausmann retrouve le genre dramatique avec plus de sérénité dans la "farce intersidérale " Palissandre et Mélasse, texte aussi curieux que politiquement brûlant, du moins à l'époque de sa composition. L'on ne saurait mieux en présenter le contenu que l'auteur lui-même, dans une lettre du 21 janvier 1960 à Achille Perilli, éditeur de la revue italienne l'Esperienza Moderna:

C'est le drame d'un morceau de palissandre sur lequel on a posé un melon. Après quelques vains essais du vieux bois pour obtenir l'amour du melon, il se produit une explosion nucléaire qui les transporte sur la Lune, où, en outre, il n'y a pas d'autres habitants que d'énormes cafards; Palissandre, redevenu arbuste, trouve que tout est comme sur la Terre et que certaines «lois » de la physique terrestre étaient fausses ; [il trouve qu'il] a, par le choc, changé de sexe, et même qu'il est enceint de Mélasse. Il (ou Elle) met au monde un nouveau melon. À cet instant arrive un engin interstellaire russe, les hommes descendent et se déshabillent à cause de la chaleur. Ils ont des cœurs automatiques. L'un d'eux s'approche de Palissandre, car il découvre une plante terrestre, et il [enroule] les fils électriques de son cœur, qui sont attachés à des batteries à sa jambe, [autour de] l'arbuste de Palissandre. Finalement Palissandre doit suivre les Russes et revient sur la Terre, mais son fils, l'autre melon, reste sur la Lune.

Le tout comprend seize pages et je pensais l'illustrer avec des photogrammes et des photopictogrammes. Je crois que cela serait un morceau de littérature vraiment contemporaine ${ }^{21}$.

17 Le «drame» existe en plusieurs versions plus ou moins élaborées, en français et en allemand, soit pièce en un acte, soit pièce radiophonique, le manuscrit initial faisant défaut comme d'ailleurs la plupart des manuscrits de textes d'une certaine longueur ${ }^{22}$.

Le jeu de mot du titre, cette allusion évidente à Pelléas et Mélisande de Maurice Maeterlinck, tragédie symboliste par excellence adaptée à l'opéra par Claude Debussy, refuse le modèle littéraire sous forme de citation détournée. Au persiflage du titre s'ajoute la mise en garde du lecteur ou spectateur dans la deuxième préface : «Cette pièce de théâtre n'est pas une pièce de théâtre ${ }^{23}$ » puisque sur la Lune manqueraient les ingrédients indispensables à ce genre : « l'action, les faits changeants, le dialogue ${ }^{24}$ » Vu ce concept anti-dramatique, l'on n'est pas bien loin du théâtre absurde d'un Beckett ou d'un Ionesco. Hausmann lui-même se réclame expressément d'Alfred Jarry, précurseur du dadaïsme et inventeur de la pataphysique, en appliquant à plusieurs reprises l'épithète « jarridique » à son drame interstellaire.

19 Une fois de plus, l'ex-dadaïste berlinois recourt dans Palissandre et Mélasse aux chevaux de bataille de la satire, de l'ironie, de la parodie, pour traiter de sujets politiques et sociaux d'actualité. Derrière le scénario "lunatique ", sous le comique de situation et de mots, étonnamment plus réussi dans la version française que dans le texte allemand, se dissimulent l'appréhension d'une guerre nucléaire et le rejet de la course aux armements de l'Union soviétique et des États-Unis ${ }^{25}$. Cette angoisse latente devient manifeste dans son «film choc» L'Homme qui a peur des bombes (1957), où Hausmann la mime et la transmet avec une puissance expressive surprenante, à l'échelle de la Danse macabre de Camille Saint-Saëns servant de musique de fond: «Jamais nous n'oublierons les traits 
hallucinés de ce traqué aux prises avec l'horreur des bombardements", s'effraie un critique $^{26}$.

En 1957 paraît également un petit livre de dialogues sous le titre de Traité de questions sans solutions importantes ${ }^{27}$. Si ce Traité ne donne, comme l'indique clairement son titre, aucune solution valable, il ne pose aucune question véritable non plus. L'auteur lui-même le qualifie de "petite farce ${ }^{28}$ » et le range ainsi sous l'étiquette d'un genre littéraire de comique grossier. Les onze dialogues entre deux personnes anonymes A et B partent d'une locution courante ou d'un proverbe plus ou moins connu - sentant parfois la traduction de l'allemand - et semblent effectivement insensés et cocasses à première vue. Et pourtant, les entretiens sur les sujets tels que "Où il y a de la fumée, il y a du feu ", "Qui marche longtemps va loin» ou encore «Si le coq chante sur le fumier, le temps change ou reste comme il est", obéissent à une vérité déroutante et donnent "l'illustration d'un non-sens absolu, d'où parfois émergent des propositions d'une implacable et inquiétante logique ${ }^{29} »$. André Weber, journaliste et ami de l'auteur, résume le « savoureux essai » en termes convaincants :

Sous forme de petits dialogues incisifs et de propos badins, Raoul Hausmann qui manie l'ironie avec la virtuosité d'un acrobate, s'interroge sur l'origine des trous dans le fromage, par exemple. Partant des dictons classiques, relevant de l'Almanach Vermot, il en tire la quintessence expressive. Humoriste à froid, Raoul Hausmann est aussi un penseur vertigineux, à qui le don de voyance et l'art de l'illustration [ne] sont pas interdits ${ }^{30}$.

21 L'Avis qui précède les échanges étranges entre les interlocuteurs est indubitablement animé par l'esprit dada -abrégé en « DD » - et mène le lecteur littéralement par le bout du nez :

Le lecteur qui prend en main (il ne peut pas prendre par les pieds) ce Traité de questions sans solutions importantes doit, sans question, s'étonner que l'auteur ait voulu chercher des solutions à des questions importantes. Il faut alors remarquer (qui? le lecteur ou l'auteur) que toutes nos notions en matière de réflexion lumineuse ou lucide dérivent de la grande école de déductions importantes que l'on appelle NEZ (ou est-ce du ZN, ou DD ?).

L'allusion à la philosophie Zen semble évidente ; or, c'est aussi à l'homme Zend-Avesta qu'il faut penser, concept du chercheur, psychologue et philosophe allemand Gustav Theodor Fechner (1801-1887), que l'on retrouve dans d'autres textes de Hausmann. Il entend par là un être qui participe à la conscience collective de l'univers entier. À l'opposé de cet homme exceptionnel ayant retrouvé le Urgrund, la cause primordiale, les deux interlocuteurs du Traité n'y parviennent décidément pas.

Ce n'est qu'à la fin de sa vie que Hausmann publie ses œuvres majeures en langue française, les essais de La Sensorialité Excentrique ${ }^{31}$ en 1970, et Sagemorcim ${ }^{32}$ en 1971, paru peu après la mort de son auteur. Ce dernier ouvrage poétique est loin d'être une œuvre de "maturation" au sens propre du terme, comme la classifie Jean Mazeaufroid ${ }^{33}$. Il réunit en fait des textes de 1946 à 1970, comprimant ainsi un travail de longue haleine sur la mythologie et transmuant en poésie les investigations mytho-critiques que l'artistechercheur entame dès le début des années quarante.

"C'est la sagesse suprême de la mort qui se cache dans le mot, séparé, retourné à double sens, car le sens est tout petit et très grand à la fois [...] le multidimensionnel Micromégas » : par ces propos sur lesquels s'ouvre le texte, Sagemorcim se révèle être un anagramme de Micromégas, conte philosophique et fantaisiste de Voltaire publié en 1752. Conjointement, le titre associe les deux concepts de «sagesse » et de «mort» dont il 
s'agit de reconnaitre le lien fondamental. Pour Hausmann, la «sagesse suprême de la mort » consiste à retrouver, grâce à la poésie, le principe essentiel de l'existence, antagonisme et unité à la fois. Le long poème central L'immortellemorte est l'illustration la plus pure de la transcendance de la mort par une poésie que Hausmann définit plus loin comme " la Poésie "ÊTRE" / la poésie Présente ». Cette poésie, apte à nous amener " aux frontières de la folie ", refuse toute compréhension rationnelle en faveur d'une sensibilité qui nous laisse deviner, par intuition, les phénomènes secrets de la vie. En ce sens seulement, Sagemorcim peut être considéré comme une sorte de «testament poétique, où Hausmann a voulu condenser en peu de pages, sa conception de l'existence et de la vie en poésie ${ }^{34} »$.

Tout au long de Sagemorcim s'opère une imbrication phonétique complexe et exigeante de thèmes scientifiques et mythologiques, autobiographiques et psychanalytiques. Les cinq sous-chapitres de Thèbes ou la sanction pragmatique, agencés autour des personnages mythiques de Jocaste et d'Oedipe opposent femme et homme, matriarcat et patriarcat, hyle et eidos, matière et esprit, et sont en débat intertextuel avec les écrits de Johann Jakob Bachofen à qui Hausmann consacre également des essais. Aussi bien cette série de dialogues que le texte annexe Thèbes Acte I rappellent d'autres noms et traditions encore : Sophocle et Eschyle, Racine et Hölderlin, sur les traces desquels Hausmann projetait de créer une tragédie "moderne » intitulée Thébaïde, afin de lutter par l'intermédiaire de l'art contre la technique et la science actuelles. La rencontre entre Oedipe et le Sphinx mise en scène dans Thèbes Acte I fait en outre pendant à un article allemand de Hausmann sur la transition du matriarcat au patriarcat exprimée dans le mythe d'œEdipe ${ }^{35}$. Dans l'image du Sphinx, gardien des lois chthoniennes, vieux serpent qui naît, meurt et renaît, " signe de sagesse ", et plus encore dans le symbole des deux serpents entrelacés, témoin de la fusion entre le mâle et la femelle, l'on approche de la quintessence de Sagemorcim.

Revenons à présent aux archives de l'artiste sur lesquelles nous avons travaillé une dizaine d'années et qui valent la peine d'être présentées plus en détail.

"As-tu pensé à ce que deviendront après toi tes documents, les programmes des matinées et soirées Dada de Berlin et autres ${ }^{36}$ ? » À cette question qu'Yves Poupard-Lieussou pose à Raoul Hausmann, dont il établit la première biobibliographie, la réponse affirmative ou négative reste difficile à donner a posteriori. Certes, le «dadasophe officiel » était loin d'être l'archiviste de lui-même, comme Goethe, par exemple, mais il n'aimait pas non plus le chaos total. Il tenait en effet des cahiers de textes et des chemises de correspondances, sans doute à l'aide des deux femmes avec lesquelles il vivait à Limoges depuis 1944, Hedwig Hausmann, née Mankiewitz, sa seconde épouse, et Marthe Prévôt, sa compagne. Des agendas annuels nous montrent également un certain souci de ne pas perdre le fil dans ses activités multiples, dans sa correspondance qu'il pratiquait quasi quotidiennement, et plus prosaïquement, dans ses revenus et dépenses.

Les archives Raoul Hausmann du MAC se composent, à vrai dire, d'un certain nombre de "sous-fonds", que nous nous contentons d'énumérer brièvement et sommairement, avant de présenter plus en détail le volet littéraire, poétique et théorique, de même que quelques bijoux, publiés entre-temps ou encore inédits.

Grosso modo, le fonds est structuré en trois grands domaines : il y a d'abord les œuvres plastiques, les tableaux, les photographies - tirages et négatifs -, les feutres, les dessins ; puis les textes, les lettres, les cahiers, les agendas, les revues, la bibliothèque; enfin les cravates, les appareils photographiques, la machine à écrire ${ }^{37}$. 
ologiquement, le fonds français couvre la période d'après-guerre, de 1945 à 1971 alors que le fonds allemand, conservé à la Berlinische Galerie, se constitue à partir de 1900 jusqu'en 1933, date de l'émigration de Hausmann de l'Allemagne nazie ${ }^{38}$. Pendant l'exil, de 1933 à 1944, avant son installation définitive à Limoges, Hausmann n'était pas inactif, bien au contraire ; il travailla, entre autres, sur la seconde partie de son roman Hyle, réalisa des études sur l'architecture rurale à Ibiza, publia des articles sur la photographie $^{39}$. Au vu de ses pérégrinations qui le mènent, en dix ans à peine, d'Ibiza à Paris et illico presto de Paris à Ibiza, d'Ibiza à Prague via Zurich, de la Tchécoslovaquie en France via la Suisse, de Paris à Peyrat-le-Château dans le département de la HauteVienne, et enfin dans la capitale limousine, on peut supposer quelques pertes et égarements de documents, manuscrits et matériaux.

Le sous-fonds littéraire du MAC, comptant quelques 10000 feuilles à lui seul, en plus des 6700 pages environ de la correspondance, peut être décrit selon plusieurs critères, linguistiques, thématiques et génériques, au sens large du terme - inutile de répéter ici les réflexions et résultats de l'inventaire et du premier cahier Hausmann. Écrits sur Dada et Néo-dada, recherches sur l'optophonétique et la photographie, textes théoriques et essais divers, textes poétiques variés, livres publiés et en chantier, en sont les dossiers structurants. Linguistiquement, l'allemand et le français prédominent ; quelques versions sont également en anglais.

30 Cinq textes majeurs en langue allemande du fonds furent publiés de 1994 à 2006, dont nous évoquons rapidement les titres :

- Aussichten oder Ende des Neodadaismus (Chances ou fin du néo-dadaïsme) ${ }^{40}$;

- Die exzentrische Empfindung (La Sensorialité excentrique) ${ }^{41}$;

- Umbruch (Bouleversement) ${ }^{42}$;

- Hyle. Ein Traumsein in Spanien (Hyle. Un état-rêve en Espagne) ${ }^{43}$;

- Hans/Jean Arp ${ }^{44}$.

$31 \mathrm{Au}$ lieu de les commenter individuellement, nous nous bornerons ici à rappeler quelques choix éditoriaux fondamentaux, qui concernent toutes nos éditions de livres et de textes épars de Hausmann jusqu'à présent. En effet, loin de vouloir prétendre à des éditions critiques, nous avons renoncé d'emblée à la pratique qui consisterait à surcharger les textes par des annotations trop nombreuses et trop détaillées pour proposer des versions authentiques et lisibles (Leseausgaben en allemand), accessibles à un public plus large et sollicitant davantage la participation du lecteur, qui peut ainsi découvrir à sa guise la vivacité et l'originalité de l'écriture de Hausmann et en inférer du sens de manière autonome. Là aussi, les critères du bien- et du mal écrire sont difficiles à appliquer tant il est vrai que le style « dadasophique » est à la fois désinvolte et déconcertant. La décision fut prise d'un commun accord avec les éditeurs Droschl, Haymon et Belleville, qui voulaient d'abord créer un public pour Hausmann avant de s'aventurer dans des projets plus amples.

Absence d'appareil critique ne signifie pas pour autant absence d'ambition scientifique. Chacune de nos éditions - à l'exception de Aussichten oder Ende des Neodadaismus (Chances ou fin du néo-dadaïsme) qui est une reproduction en fac-similé - comprend une notice éditoriale dans laquelle nous expliquons, entre autres, comment et pourquoi telle ou telle variante de texte a été sélectionnée et si, oui ou non, des modifications ont été apportées. Cette notice s'insère à chaque fois dans une postface circonstanciée, retraçant la genèse du texte, ses particularités et visées. 

œuvres originales de Hausmann, ne serait-ce que par un petit dessin sur la couverture comme Umbruch (Bouleversement). Là aussi, Chances ou fin du néo-dadaïsme sort du cadre, mais le livre que cet essai complète, Je suis toutefois le plus grand expérimentateur d'Autriche, compense largement ce manque puisqu'il est, lui, abondamment illustré. Autant que possible, le choix des illustrations rejoignait les indications que Hausmann a données à ce sujet.

Le troisième dénominateur commun de nos éditions réside dans le fait que, sans conteste, Hausmann lui-même avait envisagé la publication de ces textes. Certes, ce n'est pas un critère éditorial absolu. Pensons tout simplement au cas de Franz Kafka ${ }^{45}$ - ou à l'anthologie de textes poétiques rassemblés dans le second Cahier Raoul Hausmann, qui y déroge sans scrupules -, mais le respect de cette volonté permettait aussi de remédier enfin, indirectement et tardivement, à de nombreuses tentatives frustrantes pour l'auteur de trouver un éditeur. À travers ses lettres de supplique et de plainte adressées à différentes maisons d'édition et leurs responsables, au vu de pages entières supprimées à la demande de tel ou tel rédacteur, à la lumière de tout ce qui est resté inédit, on mesure la déception que l'ancien dadaïste a dû ressentir sans pour autant baisser les bras.

S'il est vrai que les pièces maitresses en langue allemande du fonds littéraire de Hausmann, conservé au MAC, ont été publiées - quelques beaux textes l'ont été également en français ${ }^{46}$-, il n'en demeure pas moins que, d'une part, toutes les éditions ne sont pas encore traduites ni même en cours de l'être, et d'autre part, il reste encore de véritables inédits, par exemple les essais sur la photographie infrarouge, sur l'île d'Ibiza, sur l'histoire de l'art et la mythologie. De taille variable, de qualité inégale et de finition plus ou moins aboutie, ces textes se prêteraient fort bien également à des études à part entière. Signalons enfin que des éditions critiques nous semblent malgré tout aussi nécessaires que fastidieuses, vu la complexité du fonds d'une part, les hésitations éditoriales de l'autre. Sans doute, certains desiderata de la recherche ne seront-ils pas réalisables sans des recherches pluridisciplinaires, plurilingues et internationales.

Parmi les écrits inédits d'une certaine ampleur, dont le fonds dispose intégralement, citons Ibiza - eine vergessene Insel (Ibiza - une île oubliée). Le "dossier génétique " ${ }^{47}$ comprend plus de 160 feuilles dactylographiées et manuscrites, des croquis et des documents annexes.

«Si Hausmann continue à être "Dadasophe" à Ibiza, il est aussi anthropologue, ethnologue, archéologue, architecte, mais aussi poète et photographe ${ }^{48}$. Ce constat de Barthomeu Marí se confirme sans conteste à l'examen du fonds du MAC. Or, les écrits sur Ibiza, et cela est passé inaperçu jusqu'ici, s'inscrivent dans une optique beaucoup plus large, à savoir celle d'une histoire de l'art populaire européen dans toutes ses dimensions et expressions : architecture, peinture, sculpture, outils et coutumes. L'idée de ce vaste projet de livre naît vers 1937 à Prague où Hausmann essaie de monter une exposition internationale sur les sources psycho-morphologiques de l'architecture rurale en Europe. Parallèlement, il envisage de fonder d'une part le groupe Culture européenne, d'autre part un Institut pour l'exploration morphologique de l'architecture.

Abandon ou échec de projets, perte ou disparition de sources, nous sommes encore réduits à des hypothèses et spéculations. La transcription intégrale des agendas et cahiers de textes apportera-t-elle de nouveaux éclairages? 
Car il existe bel et bien des manuscrits qui attendent encore leur exploitation. Il s'agit d'un ensemble conséquent d'une trentaine de cahiers de textes que l'auteur tenait de 1945 à 1970.

À vrai dire, Hausmann attachait peu d'importance à son "écriture authentique ${ }^{49}$ ", comme il l'explique dans une lettre à son amie Doris Hahn, et il considérait ses manuscrits comme des brouillons qu'il jetait souvent. C'est le texte mis au propre, le tapuscrit, la dactylographie, qui comptait pour lui, et il $\mathrm{y}$ a effectivement très peu de manuscrits autographes, la majorité des documents étant des tapuscrits tapés à la machine à écrire.

Les manuscrits se présentent donc sous forme de cahiers de textes, à spirales depuis 1955, le plus souvent quadrillés de la marque Clairefontaine, dimensions $22 \times 17 \mathrm{~cm}$, de la marque Herakles en 1968-69. Hausmann avait l'habitude de remplir d'abord les pages de droite avant de tourner le cahier pour commencer dans l'autre sens. Le mécanisme de l'inversion n'anime pas seulement le jeu entre le sens et le non-sens de ses poèmes, mais imprègne sa façon même d'organiser matériellement son écriture. Parfois, sur une seule feuille, les deux directions de l'écriture se rejoignent et se superposent, les langues se mélangent. De surcroît, les cahiers utilisés après 1965 perdent de leur lisibilité au fur et à mesure que la vue de l'auteur se dégrade. Dans un poème illisible, barré amplement à la main mais dont le titre reste déchiffrable, cette tendance, subie ou voulue, à l'illisibilité atteint son paroxysme.

40 Somme toute, les manuscrits et particulièrement les cahiers de textes se situent à la lisière des grands dossiers en chantier (Hyle, Ibiza, la mythologie), car ils sont des ensembles hybrides qui comprennent aussi bien des textes de tous genres, des lettres, dessins, croquis, comptes et calculs, notices, observations, citations. Ils ressemblent à une sorte de carrière d'où l'on peut extraire une multitude d'informations sur l'artiste et son œuvre. Leur étude alimente notre inventaire et tous nos commentaires de textes jusqu'à présent, leur richesse reste cependant à exploiter à fond.

41 «Quand sera-t-on enfin suffisamment lucide dans l'espace germanophone pour publier vos œuvres complètes ${ }^{50}$ ?» demande Friedericke Mayröcker dans une lettre à Raoul Hausmann, et la " princesse de la poésie autrichienne », comme il aime l'honorer, ajoute «je trouve cela plus nécessaire que toute autre chose ${ }^{51} »-$ nous aussi. Le «maître ", tel qu'il est vénéré par Mayröcker et sa génération- Ernst Jandl et le Groupe de Vienne surtout -, répond sur un ton sceptique : « Une édition de toutes mes œuvres? Et bien, cela atteindrait une grande ampleur ${ }^{52}$.» Ce défi, lui aussi, reste encore à relever, et dans les pays germanophones et en France.

\section{BIBLIOGRAPHIE}

Bartsch, Kurt, Ralf Burmeister, Adelheid Koch-Didier und Stefan Schwar, Raoul Hausmann. Biographisches und bibliographisches Lexikon, Stuttgart, Fromann-Holzboog, (Die Deutsche Literatur, VI), 2011. 


\section{NOTES}

1. Raoul Hausmannn, BUCH, Manuskripte 56, 1977, p. 57. Traduction par nos soins.

2. R. Hausmann à Walter Höllerer, 23-07-1963. Traduction par nos soins. Musée départemental d'Art contemporain de Rochechouart. Toutes les lettres et tous les textes inédits sont cités d'après les originaux conservés dans ce musée, cité par la suite par MAC.

3. Pour ma thèse de doctorat, de même que dans le cadre d'un projet de recherche (1993-1998), j'ai eu l'occasion de travailler non seulement sur l'impact de Hausmann sur la littérature autrichienne après 1945, mais aussi sur ses archives, notamment sur le fonds français conservé au MAC. Cf. Adelheid Koch, Ich bin immerhin der größte Experimentator Österreichs. Raoul Hausmann, Dada und Neodada \& ein Essay: Raoul Hausmann Aussichten oder Ende des Neodadaismus, Innsbruck, Haymon, 1994. (Version entièrement revue, élargie et richement illustrée de la thèse de doctorat Je suis toutefois le plus grand expérimentateur d'Autriche. Raoul Hausmann, Dada et Néo-dada \& un essai : Raoul Hausmann, chances ou fin du Néo-dadaïsme, Université de Graz, 1993). Du projet de recherche intitulé « Les archives Raoul Hausmann » est issu l'ouvrage suivant: R. Hausmann, Je suis l'homme de 5000 paroles et de 10000 formes. Inventaire raisonné des archives Raoul Hausmann au musée départemental de Rochechouart. Inventaire établi et annoté par Adelheid Koch-Didier avec la collaboration de Stefan Schwar, MAC, 1997.

4. Inventaire, op. cit., p. 140, 142.

5. Entretien avec Marthe Prévot. Propos recueillis par Didier Arnaudet, Raoul Hausmann, 1986-1971 (catalogue d'exposition), Mâcon éditions W, MAC, 1986, p. 55.

6. Correspondance Cahiers du Sud, MAC.

7. Lettre de Hausmann à Iliazd, 17-12-1949, MAC.

8. Richard Thieberger, Remarques préliminaires, Austriaca 19, 1984, «Écrivains autrichiens émigrés en France ", p. 9.

9. Lettre de Hausmann à Théodore Koenig, 15-09-1968, MAC.

10. Raoul passe son enfance essentiellement à Vienne et dans ses parages; à l'âge de six ans, il voyage et séjourne en Italie (Venise, Naples), puis quelques mois au Tyrol (1893) ; 1895-1896 chez ses grands-parents paternels à Cilli (Celje au sud de la capitale styrienne Graz, aujourd'hui en Slovénie) où il fréquente l'école primaire de garçons; en 1896-1897, au collège à Vienne, il commence à apprendre le français. Les souvenirs que Raoul garde de son enfance en Autriche sont mitigés : relations distanciées avec ses parents et sa sœur Mira, grand attachement au grand-père paternel; le motif romantique de l'enfance perdue sous-tend son roman autobiographique Hyle dont nous reparlerons.

11. Cf. Scharfrichter der bürgerlichen Seele. Raoul Hausmann in Berlin 1900-1933. Unveröffentlichte Briefe Texte Dokumente aus den Künstlerarchiven der Berlinischen Galerie, hrsg. und kommentiert von Eva Züchner, Berlin, Berlinische Galerie, 1998, p. 25.

12. MAC.

13. Cf. Adelheid Koch, Ich bin immerhin der größte Experimentator Österreichs, op. cit.

14. R. Hausmann, Hurrah! Hurrah! Hurrah! 12 Satiren, Berlin, Malik, 1921. Hourra! Hourra! Hourra! 12 satires politiques. Traduit de l'allemand par Catherine Wermester, Paris, Allia, 2004.

15. R. Hausmann, Dadasophie, Phantomas, 1967, nº 68-72, p. 59-60.

16. Inventaire, op. cit., p. 117.

17. Ibid., p. 48.

18. Alfred Kolleritsch, Raoul Hausmann, marginalie, manuskripte 6, 1966, $\mathrm{n}^{\circ} 16$, p. 1 .

19. Hausmann adopte la nationalité tchèque en 1919 et divorce la même année d'avec sa première femme, la violoniste berlinoise Elfriede Schaeffer. En 1923, il épouse Hedwig Mankiewitz, fille d'un banquier juif de Berlin, qui, elle aussi, optera pour la nationalité tchèque. 
Pendant des années, Hausmann voyage avec un passeport tchèque qui n'aura plus cours à l'issue de la Seconde Guerre mondiale à cause de la langue maternelle allemande de son détenteur : le 17 janvier 1951, la section consulaire de l'ambassade de la République tchécoslovaque à Paris certifie que le couple Hausmann a perdu la nationalité tchèque en vertu de la loi constitutionnelle tchécoslovaque du 2 août 1945. Devenu apatride et placé sous la protection administrative de l'Organisation Internationale pour les Réfugiés, Hausmann tente en vain d'obtenir d'abord la nationalité autrichienne, puis française. En juin 1959, lorsqu'il dépose sa demande de naturalisation auprès de la préfecture de la Haute-Vienne, il a soixante-treize ans. Malgré le soutien et les recommandations de personnages influents au ministère de la Santé publique et de la Population de même qu'au ministère de la Culture (parmi lesquels André Malraux, à l'époque ministre d'État chargé des affaires culturelles), son âge avancé constitue « le principal, pour ne pas dire l'unique obstacle » à son " plus cher désir », comme il le formule dans une lettre du 17 mai 1961, de «devenir sujet français » (lettres de Noël Arnaud à Hausmann, 27-11-1959 et de Hausmann, sans destinataire, 17-05-1961, MAC). Au bout de trois ans de requête infructueuse, Hausmann finit en 1961 par accepter malgré lui la nationalité allemande - acquise en vertu d'un décret sur l'appartenance des anciens citoyens tchécoslovaques de langue allemande à la République fédérale d'Allemagne - pour mettre fin aux inconvénients liés à son statut d'apatride en France.

20. Cf. La Poésie a pour objet le MOT: Raoul Hausmann, écrivain, présenté par A. Koch-Didier, MAC, 1997, (Cahiers Raoul Hausmann, 1).

21. Lettre de Hausmann à Achille Perilli, 21-01-1960, MAC.

22. Le texte français date de 1957-1958, un extrait parut dans Phantomas, 1967, $\mathrm{n}^{\circ}$ 68-72, p. 21-42. Une version allemande, elle aussi réduite, fut publiée dans manuskripte 13, 1973, nº 40, p. 22-26. Nous avons publié le texte intégral français dans le $2^{\mathrm{e}}$ cahier Raoul Hausmann en 1999. Cf. Kaléidoscope. Choix de textes poétiques de Raoul Hausmann, édition établie et préfacée par Adelheid Koch-Didier, MAC, 1998, (Cahiers Raoul Hausmann, 2.), p. 5-29.

23. R. Hausmann, Palissandre et Mélasse, deuxième préface, tapuscrit, p. 2.

24. Ibid.

25. Cf. Hausmann à Augustin Tschinkel, 26-8-1966, MAC.

26. La pantomime fut jouée pour la première fois chez Jean Arp à Meudon, plus tard à Paris ( $c f$. Hausmann à Arp, 05-07 et 21-07-1959) ; la critique citée se trouve parmi les coupures de journaux, datée du 24 juin 1957, signée A. W. (André Weber), MAC.

27. R. Hausmann, Traité de questions sans solutions importantes, Bâle, Éditions Panderma/Carl Laszlo, 1957.

28. Lettre à Maud Westerdahl, 14-03-1966, MAC.

29. Jean Mazeaufroid, L'itinéraire poétique de Raoul Hausmann (1886-1971), mémoire de maîtrise, Limoges 1973, p. 67.

30. André Weber, Ironie dadaïste. Cité d'après le dossier de coupures de journaux de Hausmann, sans indication du nom du journal, rubrique Arts-lettres et spectacles, 22-02-1958, MAC.

31. R. Hausmann, La Sensorialité excentrique, édition originale français/anglais, Cambridge, Ormiston 1970. (collection OU.) Nouvelle édition français/allemand, avec des illustrations de R. Hausmann, édition établie et postface par A. Koch, Graz, Wien, Literaturverlag Droschl, edition neue texte, 1994.

32. R. Hausmann, Sagemorcim, Bruxelles, Henri Fagne, 1971.

33. J. Mazeaufroid, Itinéraire poétique, op.cit., p. 74.

34. Ibid.

35. Cf. R. Hausmann, Oedipus, eine Wende im alteuropäischen Bewußtsein, inventaire, op. cit., p. $74,226$.

36. Copie d'une lettre de Poupard-Lieussou, jointe à une lettre de Hausmann à Hans Richter du 9 décembre 1964, MAC. 
37. Aux archives du MAC figurent, entre autres, des ensembles complets concernant Ibiza et la mer Baltique, de même que les photomontages et photo(picto)grammes que Hausmann réalisa après la Seconde Guerre mondiale. Cf. le site du MAC qui donne les précisions suivantes : «Depuis 1984, est constitué au musée départemental de Rochechouart un ensemble représentatif d'œuvres de Raoul Hausmann. Le fonds conserve 180 œuvres (collages, photographies, peintures, dessins, impressions) et 254 feutres ainsi qu'un fonds d'archives constitué de textes (813), de la correspondance (environ 6000 lettres) et des négatifs (environ 1600 ). De plus, le fonds possède des matériaux complémentaires comme les enregistrements de poèmes phonétiques et la bibliothèque de l'artiste avec des livres provenant de tous les lieux où il a vécu: Berlin, Ibiza, Zurich, Paris, Prague, Limoges. En 2002, une nouvelle acquisition a apporté au fonds 256 œuvres et ébauches ainsi que 223 contacts (tirage d'époque, d'un petit format). (750 livres, catalogues et revues)».

http://www.musee-rochechouart.com/hausmann_sommaire.htm

38. Des documents antérieurs au déménagement de la famille Hausmann de Vienne à Berlin en 1900 se trouvent également à Berlin; $c f$. l'édition critique du fonds berlinois, Scharfrichter der bürgerlichen Seele, op. cit.

39. Notamment dans la revue suisse Camera, Luzern, 1934.

40. Reproduit en fac-similé in A. Koch, Experimentator,(Expérimentateur) op. cit., p. 227 sq.

41. R. Hausmann, Die exzentrische Empfindung, (La Sensorialité excentrique) op. cit.

42. R. Hausmann, Umbruch, (Bouleversement), hrsg. und mit einem Nachwort von A. Koch (édité et postface par A. Koch), Innsbruck, Haymon, 1997.

43. R. Hausmann, Hyle. Ein Traumsein in Spanien, (Hyle. Un état-rêve en Espagne), hrsg. und mit einem Nachwort von A. Koch-Didier, (édité et postface par A. Koch), München, Belleville, 2003. Première édition Frankfurt/M., Heinrich Heine, 1969.

44. R. Hausmann, Hans/Jean Arp, hrsg. von A. Koch-Didier, München, Belleville, 2008. L'édition est illustrée de photographies, parmi lesquelles les prises de vue que Hausmann a réalisées d'Arp en 1938, et d'autres réalisées par Marthe Prévot à la demeure d'Arp à Meudon.

45. Rappelons que Kafka (1883-1924) ne publia que très peu de textes de son vivant et qu'il chargea son ami et exécuteur testamentaire Max Brod de brûler tous ses manuscrits après sa mort. Brod ne procédera pas à ses dernières volontés et publiera les grands romans dès les années 1920.

46. Cf. Kaléidoscope, op. cit. ; cf. aussi R. Hausmann, Palissandre et Mélasse, Dada circuit total, dossier coordonné par Henri Béhar et Catherine Dufour, Lausanne, L’Âge d'Homme, 2005, (Les Dossiers H), p. 589-599. Une anthologie de textes poétiques comprend aussi quelques inédits, tout en étant, à notre avis, assez contestable sur le plan scientifique. Cf. R. Hausmann, Une anthologie poétique, précédé de RH l'optophonétiste par Isabelle Maunet-Salliet, livre et $\mathrm{CD}$, Paris, Al Dante/ Transbordeurs, 2007. Cf. notre compte rendu, Austriaca n 64, 2007, p. 207-211.

47. Un « dossier génétique » peut « inclure des lettres, des entretiens, des témoignages extérieurs etc. » Philippe Lejeune, «Génétique et autobiographie », Lalies n²8, éditions rue d’Ulm, Presses de l'École Normale Supérieure, 2008.

48. Barthomeu Marí, préface au catalogue Raoul Hausmann, architecte - architect Ibiza 1933-1936, Bruxelles, Fondation pour l'architecture, 1990. Dans ce catalogue bilingue, le séjour à Ibiza et les œuvres qui en résultent sont examinés sous plusieurs angles: histoire de l'art, architecture, contexte biographique, milieu culturel et traditions rurales, ruptures et cohérences.

49. Lettre du 17-22-03-1967. Archives de la littérature allemande, Marbach/Neckar.

50. Lettre du 30-03-1969, MAC.

51. Ibid.

52. R. Hausmann, Briefe an Otto Breicha, Friederike Mayröcker, Briefwechsel mit Andreas Okopenko ( Lettres à Otto Breicha, Friederike Mayröcker, correspondance avec Andreas Okopenko) Grafiken vom 
Verfasser, hrsg. von Heimrad Bäcker (Illustrations de l'auteur, volume édité par Heimrad Bäcker), Sondernummer neue texte, 1979, 36/37, p. 12.

\section{RÉSUMÉS}

Co-fondateur du dadaïsme à Berlin, Raoul Hausmann (1886-1971) est encore trop peu perçu comme écrivain de langue française. Ses archives conservées au musée d'Art contemporain de Rochechouart permettent cependant de découvrir cet artiste protéiforme qui fut peintre, photographe, poète, romancier, théoricien et danseur. Notre article se propose de retracer le lien entre le multilinguisme de Hausmann et sa créativité d'écrivain qui s'obstine à s'affranchir de toutes les normes linguistiques et artistiques.

Raoul Hausmann (1886-1971), who was a co-founder of the Berlin Dada movement, is still too rarely seen as a French-speaking author nowadays. Yet his archives, which are kept in the Musée d'Art contemporain (Contemporary Art Museum) in Rochechouart, grant us a comprehensive insight into this versatile artist who was a painter, a photographer, a poet, a novelist, a theoretician and a dancer. My article aims to study the link between Hausmann's multilingualism and his writing creativity through which he attempted to free himself from all linguistic and artistic norms. 